\title{
What Has Changed During the COVID-19 Pandemic? - The Effect on an Academic Breast Department in Portugal
}

\author{
(D) Diana Simão, (D) Mariana Sardinha, (D) Ana Filipa Reis, (iD Ana Sofia Spencer, (D) Ricardo Luz, (ID Sónia Oliveira \\ Department of Medical Oncology, Centro Hospitalar Universitário Lisboa Central, Lisboa, Portugal
}

\section{ABSTRACT}

Introduction: One year ago, Portugal entered its first lockdown because of the coronavirus disease-2019 (COVID-19) pandemic. The impact of this on delays in cancer diagnosis and treatment is a major concern, which may negatively affect the outcomes of these patients.

Materials and Methods: This retrospective, single-center analysis compared the clinical and pathological characteristics of breast cancer (BC) patients referred to a medical oncology first appointment between March 2020 and 2021, with the same period in the previous year.

Results: Strikingly, there was a $40 \%$ reduction in the number of BC patients during lockdown. However, there was a statistically significant increase in the proportion of metastatic BC patients admitted for the first time for systemic therapy $(13.6 \%$ vs. $28.9 \%, p=0.003)$. Additionally, a statistically significant increase in the number of patients with bilateral early BC at diagnosis after March 2020 was found $(7.2 \%$ vs. $1.9 \%, \mathrm{p}=0.043)$.

Conclusion: These findings support international recommendations for an accelerated restoration of BC screening, to reduce incidence of advanced breast cancer at diagnosis and mitigate the expected impact of the COVID-19 pandemic on patients with cancer. Further work is needed to examine in detail the impact of measures to manage the COVID-19 pandemic on breast cancer outcomes.
\end{abstract}

Keywords: COVID-19, SARS-CoV-2, breast cancer, oncology department, tertiary hospital

Cite this article as: Simão D, Sardinha M, Reis AF, Spencer AS, Luz R, Oliveira S. What Has Changed During the COVID-19 Pandemic? - The Effect on an Academic Breast Department in Portugal. Eur J Breast Health 2022; 18(1): 74-78

\section{Key Points}

- One year of COVID-19 pandemic: effects on a breast cancer oncology department.

- Increase of metastatic breast cancer patients admitted for systemic therapy.

\section{Introduction}

The severe acute respiratory syndrome coronavirus 2 (SARS-CoV-2) associated disease (COVID-19), first recognized in Wuhan, in December 2019, was declared by the World Health Organization (WHO) as a global pandemic on 11th March 2020 (1). On March 2nd, 2020, the first COVID-19 cases were confirmed in Portugal. Only sixteen days later, 642 cases and three deaths were reported. The Portuguese authorities took early action to control the COVID-19 outbreak, initiating lockdown measures and declaring a state of emergency on March 18th, 2020 (2). By the beginning of April 2020, over 1 million cases of confirmed infections and over 60 thousand deaths from COVID-19 were reported worldwide. Over the last year the COVID-19 pandemic has led to more than 100 million cases and 2.8 million deaths worldwide (3).

Older age and comorbid disease, such as cancer, have been identified as potential risk factors for poor prognosis in COVID-19, including the need for intensive care, invasive ventilation, and death (4). Despite data that suggest that COVID-19 is typically more severe and lethal among people with underlying medical conditions, including active cancer, such risk may be influenced by the type of cancer, treatment, time since treatment, patient age and comorbid medical conditions (5-7).

Moreover, the COVID-19 pandemic represents a global challenge, not only for reorganization of health care resources in order to minimize exposure risks, especially in oncology settings, but also in ensuring the continuity of care during cancer diagnosis and treatment (8). 
International guidelines have been published to guide patients and healthcare professionals for the prevention and management of COVID-19 in order to maximize the available resources. The European Society for Medical Oncology (ESMO) has established guidance for clinicians, defining levels of priorities regarding medical interventions, based on the ESMO Magnitude of Clinical Benefit Scale (MCBS), a public health tool intended to support the uptake of medical interventions in oncology (9). In parallel, local and national guidelines were published (10). The Portuguese Society of Oncology (SPO) published recommendations that were taken into account by the Portuguese health authorities to issue a standard of action in the provision of care to cancer patients, in April 2020 (11).

Despite cancer centers/departments continuing to function, the imposition of the national lockdown resulted in a reduction in the numbers of patients accessing healthcare. Delay in cancer diagnosis and treatment due to the COVID-19 pandemic is a major concern, but the true impact is not yet clearly established (12-14).

Although breast cancer (BC) is one of the most frequent cancers and represents the leading cause of oncological death among women worldwide, there has been an improvement in terms of prognosis over the last 20-30 years. The significant gains were largely attributable to early detection and systemic therapies. ESMO recommendations prioritized highest risk $\mathrm{BC}$, in accordance with current clinical practice, to maintain improved survival (15).

The scale of the diagnostic and treatment delay attributed to the pandemic and whether it is equally distributed is currently unknown. Using data from a single center, our study aimed to evaluate the consequences of the pandemic on the referral of patients with $\mathrm{BC}$ to the medical oncology unit, compared with the previous year.

\section{Materials and Methods}

The purpose of this non-experimental, descriptive, retrospective, single-institution analysis was to evaluate the impact of the COVID-19 pandemic on the admission demographics and characteristics of BC patients between March 2020 and March 2021, compared to the same period one year previously. BC patients were evaluated who were referred to the medical oncology department after multidisciplinary board discussion, which had been taking place virtually since April 2020 .

Clinical records were used for the data collection for each patient. Baseline demographic information included sex, age, Eastern Cooperative Oncology Group (ECOG) performance status and previous history of BC. Age at diagnosis was grouped into $<40,40$ 64 and $\geq 65$ years. Tumor characteristics included histopathology, molecular subtypes based on hormone receptor (HR) and human epidermal growth factor receptor 2 (HER2) status. HR and HER2 status were combined to form the variable "tumor subtype", as follows: HR+/HER2+; HR+/HER2-; HR-/ HER2+; and HR-/HER2-. Clinical TNM stage was defined according to the eighth edition of the American Joint Committee on Cancer (AJCC) Staging Manual.

The research was conducted ethically according to the principles of the Declaration of Helsinki. The authors confirm that patient written informed consent was obtained. Data were extracted from clinical files and is completely anonymous with no personal information being collected. The paper is exempt from ethical committee approval due to its retrospective, non-interventional nature and non-identifiable data collected.

\section{Statistical Analysis}

Descriptive statistics were generated for all data collected. For categorical variables, frequency tables were created to evaluate distributions and analyzed using Fisher's exact test (two-sided). Twotailed p values below 0.05 were considered statistically significant. Statistical analyses were performed using SPSS software, version 23 (IBM Inc., Armonk, NY, USA).

\section{Results}

The demographic, clinical and pathological characteristics of the patients included in the analyses are listed in Table 1. Between March 2020 to March 2021, 97 individuals (94 women and 3 men) were referred to the medical oncology unit for suspected BC. From March 2019 to March 2020, 162 patients were referred (160 women and 2 men), a reduction in first appointment admission of $40.1 \%$. No statistically significant difference in the distribution of ECOG performance status was observed between the two periods $(\mathrm{p}=0.366)$. Median age at diagnosis was 61 and 58 years, in 2020 and 2019 respectively, with a similar distribution of age groups $<40,40-64, \geq 65$ years in both periods $(\mathrm{p}=0.744)$.

At pathological examination in the lockdown period (2020-2021), most cases $(n=87,89.6 \%)$ were reported as invasive ductal carcinoma, while five cases $(5.2 \%)$ were lobular carcinoma and five cases $(5.2 \%)$ were defined as "others". No statistically significant differences were found with this classification from the earlier pre-lockdown period. Regarding BC biological subtypes, after March 2020, 17 patients (17.5\%) were $\mathrm{HR}+/ \mathrm{HER} 2+, 56$ (57.7\%) were $\mathrm{HR}+$ /HER2-, 5 (5.2\%) were HR-/HER2+, and 19 (19.6\%) were HR-/HER2-. No statistically significant difference in the distribution of biological features was observed between the two periods $(\mathrm{p}=0.468)$. There was a statistically significant increase in the proportion of patients with bilateral BC at diagnosis after March 2020 (7.2\% vs 1.9\%, p=0.043).

Distribution by clinical prognostic TNM staging (8th edition AJCC) is shown in Table 2. There was a decrease in the number of stage I patients, from $30.9 \%$ in 2019 to $20.6 \%$ in 2020 , however this was not significantly different $(\mathrm{p}=0.083)$. Overall, distribution of stages II-III BC did not significantly vary when compared between the two periods (stage IIA: $\mathrm{p}=0.761$; stage IIB: $\mathrm{p}=0.702$; stage III: $\mathrm{p}=1.000$ ). By contrast, after initiation of lockdown measures in Portugal, a significant increase in the proportion of patients with metastatic BC at first admission for systemic therapy was found to have increased from $13.6 \%$ in 2019 to $28.9 \%$ in 2020 ( $\mathrm{p}=0.003)$.

\section{Discussion and Conclusion}

As expected, we found that the COVID-19 pandemic had a negative effect on referral of BC patients, with a $40 \%$ reduction in first appointment admissions at the medical oncology department, compared with the previous year.

Understanding the implications of the delay in diagnosis and access to treatment for $\mathrm{BC}$ cannot be entirely captured unless contextualized to the biology of the cancer and patterns of clinical presentation, including stage and setting of care (16). Our single-institution analysis reported a significant increase in the proportion of metastatic BC patients admitted for the first time for systemic therapy after initiation 
of lockdown measures in Portugal. There was no statistically significant difference in patients with early BC. As a limitation, these interesting data may not reflect the true disruption due to COVID-19, since there was no evidence of a stage migration, but only an increase in the proportion of $\mathrm{ab}$ initio metastatic $\mathrm{BC}$. However, we also observed a significant increase in the number of patients with bilateral $\mathrm{BC}$ at diagnosis after March 2020.

This analysis only included data from a single center, located in Lisbon, which will impair the generalization of our results to other settings. However, we are currently working on increasing the patient sample with the inclusion of other centers in Portugal. This will allow a more detailed picture to emerge concerning differences in diagnosis rates or treatment strategies between regions.

The burden of COVID-19 on health systems worldwide has important implications for cancer care that we will need to address. From the onset of the lockdown, essential diagnostic services were suspended or operating at substantially reduced capacity. These suspensions were due to the risk of exposure to SARS-CoV-2 for patients and clinicians, and because of redeployment of staff towards critical care to manage patients with COVID-19. A national population-based modeling study, using English National Health Service (NHS) cancer registration estimated a 7.9-9.6\% increase in the number of deaths due to breast cancer up to year 5 after diagnosis (12).

National screening services were widely suspended from the end of March 2020, and this will have contributed significantly to the decrease in the number of early breast cancers diagnosed and treated. There has been limited data about how the pandemic affected cancer care because of screening and treatment delays. Nyante et al. (17) reported maximum reductions in March 2020 for screening and diagnostic mammography and in May 2020 for biopsies. This deficit decreased gradually, with no significant difference between observed

Table 1. Baseline demographic and pathological characteristics of patients diagnosed with invasive breast cancer and admitted in Oncology department according to year

2020-2021

97 patients
2019-2020

162 patients p-value

(\%)

\section{Gender}

Female

Male

ECOG performance status

0-1

$\geq 2$

Age at diagnosis (years)

$<40$

40-65

$>65$

Histology

Invasive ductal

Invasive lobular

Other

Subtype

HR + / HER2 -

$\mathrm{HR}+/ \mathrm{HER} 2+$

HR - / HER2 +

HR - / HER2 -

Previous history of BC

Yes

No

Bilateral BC

Yes

No
(\%) 96.9
92.8 160

2

155

21

83

58

144

12

6

98.8

1.2

95.7

4.3

13.0

51.2

35.8

88.9

7.4

3.7

61.1

19.8

1.9

17.2

28

0.468

5.6

94.4

0.099

0.366

0.562

0.744

0.699

153

3

1.9

98.1
159 
Table 2. Clinical prognostic TNM stage of patients with invasive breast cancer according to year

2020-2021

97 patients
2019-2020

162 patients p-value
(\%)
(\%)

\section{Clinical prognostic TNM stage}

I

IIA

IIB

III

IV

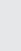

Significant associations are bolded.

TNM stage was defined according to the eighth edition of the American Joint Committee on Cancer (AJCC) Staging Manual.

TNM: tumor ( $\mathrm{T})$, nodes $(\mathrm{N})$, and metastases, n: Number and expected numbers by July and August 2020 compared with the pre pandemic population. A population-based analysis from the USA demonstrated that there was a substantial decrease in BC screening and diagnosis from March to July 2020 compared with March to July 2019 (18). For example, mammographic screening decreased by up to $85 \%$ and breast biopsies decreased by up to $71 \%$.

Similar results are reported from Europe. In England, routinely collected NHS cancer waiting time data were analyzed to compare activity for BC in the first six months of 2020 compared to the same period in 2019. The number of referrals for suspected BC was $28 \%$ lower and the number of patients who received their first treatment for a BC diagnosis was $16 \%$ lower. These data suggest that, while there was undoubtedly a marked decrease in the number of referrals made that may have led to a decrease in the numbers of newly diagnosed $\mathrm{BC}$, the magnitude of the decrease in the number of cancers was not as large as initially feared. The observed fall was proportionately much larger in patients referred non-urgently for assessment compared to those referred urgently (40\% versus 23\%) (19). A population-based study from the Netherlands showed that the incidence of BC started to decline after social lockdown and the temporary pause in screening. This decrease was seen in all age groups and all regions, compared with reference data from 2018/2019. However, the incidence of stage IV tumors did not decline. As the incidence reduction mainly occurred for the lowest stage disease, the authors suggested that the delay in diagnosis would not have had a large impact on long-term outcomes (20). A multicentric analysis from Italy, reporting the effects in the first three months after lockdown, showed a significant difference in waiting times, proportion of patients with lymph-node involvement, and cancer grading, compared with the similar period from the previous year. Nonetheless, after multivariate analysis, the significantly longer waiting time on list during the lockdown, was the only predictive factor for lymph node involvement progression (21). In a population- and registry-based study from Croatia, the average monthly percent change in referrals after the initial lockdown measures were introduced was $-11.0 \%$, resulting in a $24 \%$ reduction in newly diagnosed $\mathrm{BC}$ cases during April, May, and June compared with the same period of 2019 (22). Moreover, from the point of view of the patient, in a US national survey of BC survivors, nearly half of respondents reported delays in cancer care in the early weeks of the COVID-19 pandemic (23).
Recent multicenter analysis, including breast cancer patients, showed that chemotherapy was not associated with an increased risk of infection with SARS-CoV-2, suggesting that chemotherapy can be safely administered and should not be withheld, particularly when given for curative intent (24).

Despite available data, the real long-term impact of the pandemic on $\mathrm{BC}$ patients it is not yet known. In the near future, it will be crucial to make decisions at both institutional and national level in order to restart cancer screening and set new priorities for BC treatment.

In conclusion, one year after the first case, the COVID-19 pandemic still represents a substantial challenge in cancer care in Portugal. Our study showed a negative effect on the referral of BC patients to medical oncology, with a $40 \%$ reduction in first appointment admissions and a significant increase in these patients that did attend having metastatic BC. Further work is needed to assess the impact of measures to manage the COVID-19 pandemic on $\mathrm{BC}$ outcomes. On the other side, national authorities need to restore $\mathrm{BC}$ screening services are much as possible while taking into account the continuing pandemic to minimize cancer treatment delays.

Ethics Committee Approval: Data were extracted from clinical files and is completely anonymous with no personal information being collected. The paper is exempt from ethical committee approval due to its retrospective, noninterventional nature and non-identifiable data collected.

Informed Consent: written informed consent was obtained from the patients.

Peer-review: Externally peer-reviewed.

\section{Authorship Contributions}

Conceptualization: D.S., Methodology: D.S.; Data Collection and/or Processing: M.S., Literature Search: M.S., Analysis and/or Interpretation: A.F.R.; Visualization: A.S.S.; Writing: S.O.; Review and Editing: S.O.; Supervision: R.L.

All authors agree to be accountable for all aspects of the work and contributed to the final manuscript.

Conflict of Interest: No conflict of interest declared by the authors. 
Financial Disclosure: The authors received no financial support for the research, authorship, and/or publication of this article

\section{References}

1. WHO.int [Internet] World Health Organization virtual press conference on COVID-19. Available at: https://www.who.int/. (Date accessed: 01/04/2021). [Crossref]

2. Diário da República n. ${ }^{\circ} 55 / 2020$, 3 o Suplemento, Série I de 2020-03-18 [Internet] Decreto do Presidente da República n. ${ }^{\circ} 14-\mathrm{A} / 2020$, de 18 de março. Available at: https://www.dre.pt/. (Date accessed: 20/03/2020). [Crossref]

3. Worldometers.info [Internet] Covid-19 coronavirus pandemic. Available at: http://www.worldometers.info/coronavirus/. (Date accessed: 01/04/2021). [Crossref]

4. Liang W, Guan W, Chen R, Wang W, Li J, Xu K, et al. Cancer Patients in SARS-CoV-2 infection: a nationwide analysis in China. Lancet Oncol 2020; 21: 335-337. (PMID: 32066541) [Crossref]

5. Zhang L, Zhu F, Xie L, Wang C, Wang J, Chen R, et al. Clinical characteristics of COVID-19-infected cancer patients: A retrospective case study in three hospitals within Wuhan, China. Ann Oncol 2020; 31: 894-901. (PMID: 32224151) [Crossref]

6. Kuderer NM, Choueiri TK, Shah DP, Shyr Y, Rubinstein SM, Rivera DR, et al. Clinical impact of COVID-19 on patients with cancer (CCC19): A cohort study. Lancet 2020; 395: 1907-1918. (PMID: 32473681) [Crossref]

7. Poortmans PM, Guarneri V, Cardoso MJ. Cancer and COVID-19: What do we really know? Lancet 2020; 395: 1884-1885. (PMID: 32479827) [Crossref]

8. Tagliamento M, Lambertini M, Genova C, Barisione E, Maria AD, Grosso $\mathrm{M}$, et al. Call for ensuring cancer care continuity during COVID-19 pandemic. ESMO Open 2020; 5: e000783. (PMID: 32381594) [Crossref]

9. Curigliano G, Banerjee S, Cervantes A, Garassino M, Garrido P, Haanen J, et al. Managing cancer patients during the COVID-19 pandemic: an ESMO multidisciplinary expert consensus. Ann Oncol 2020; 31: 13201335. (PMID: 32745693) [Crossref]

10. Sociedade Portuguesa de Oncologia [Internet] Recomendaçóes para o tratamento de doentes com cancro e o COVID-19. Available at: https:// www.sponcologia.pt/ (Date accessed: 01/04/2021). [Crossref]

11. Direção-Geral da Saúde [Internet] Norma nº09/2020 de 02/04/2020. Lisboa: DGS; 2020. Available at: https://covid19.min-saude.pt/ (Date accessed: 01/04/2021). [Crossref]

12. Maringe C, Spicer J, Morris M, Purushotham A, Nolte E, Sullivan R, et al. The impact of the COVID-19 pandemic on cancer deaths due to delays in diagnosis in England, UK: a national, population-based, modelling study. Lancet Oncol 2020; 21: 1023-1034. (PMID: 32702310) [Crossref]
13. 13 Sud A, Torr B, Jones ME, Broggio J, Scott S, Loveday C, et al. Effect of delays in the 2-week-wait cancer referral pathway during the COVID-19 pandemic on cancer survival in the UK: a modelling study. Lancet Oncol 2020; 21: 1035-1044. (PMID: 32702311) [Crossref]

14. Hamilton W. Cancer diagnostic delay in the COVID-19 era: what happens next? Lancet Oncol 2020; 21: 1000-1002. (PMID: 32702312) [Crossref]

15. Azambuja E, Trapani D, Loibl S, Delaloge S, Senkus E, Criscitiello C, et al. ESMO Management and treatment adapted recommendations in the COVID-19 era: Breast Cancer. ESMO Open 2020; 5(Suppl 3): e000793. (PMID: 32439716) [Crossref]

16. Caplan L. Delay in breast cancer: implications for stage at diagnosis and survival. Front Public Health 2014 2:87. (PMID: 25121080) [Crossref]

17. Nyante SJ, Benefield TS, Kuzmiak CM, Earnhardt K, Pritchard M, Henderson LM. Population-Level Impact of Coronavirus Disease 2019 on Breast Cancer Screening and Diagnostic Procedures. Cancer 2021; 127: 2111-2121. (PMID: 33635541) [Crossref]

18. Patt D, Gordan L, Diaz M, Okon T, Grady L, Harmison M, et al. Impact of COVID-19 on cancer care: How the pandemic is delaying cancer diagnosis and treatment for American seniors. JCO Clin Cancer Inform 2020; 4: 1059-1071. (PMID: 33253013) [Crossref]

19. Gathani T, Clayton G, MacInnes E, Horgan K. The COVID-19 pandemic and impact on breast cancer diagnoses: what happened in England in the first half of 2020. Br J Cancer 2021; 124: 710-712. (PMID: 33250510) [Crossref]

20. Eijkelboom A, Munck L, Peeters M, Broeders M, Strobbe L, Bos M, et al. Impact of the COVID-19 pandemic on diagnosis, stage, and initial treatment of breast cancer in the Netherlands: a population-based study. J Hematol Oncol 2021; 14:64. (PMID: 33865430) [Crossref]

21. Vanni G, Tazzioli G, Pelliciaro M, Materazzo M, Paolo O, Cattadori F, et al. Delay in Breast Cancer Treatments During the First COVID-19 Lockdown. A Multicentric Analysis of 432 Patients. Anticancer Res 2020; 40: 7119-7125. (PMID: 33288611) [Crossref]

22. Vrdoljak E, Balja MP, Marušić Z, Avirović M, Blažičević V, Tomasovic C, et al. COVID-19 Pandemic Effects on Breast Cancer Diagnosis in Croatia: A Population- and Registry-Based Study. Oncologist 2021; 26: e1156-e1160. (PMID: 33856084) [Crossref]

23. Papautsky EL, Hamlish T. Patient reported treatment delays in breast cancer care during the COVID 19 pandemic. Breast Cancer Res Treat 2020; 184: 249-254. (PMID: 32772225) [Crossref]

24. Budhathoki N, Kucharczyk J, D’Abreo N, Kwa MJ, Plasilova M, Dhage $S$, et al. Risk for SARS-CoV-2 infection in patients with breast cancer treated with chemotherapy, biologic therapy or active surveillance: Patient outcomes from multicenter institution in New York [abstract]. J Clin Oncol 2021; 39(Suppl 15): 1513. 